\title{
Wireless Sensor Network Structural Design and Protocols: A Survey
}

\author{
Siddhi Sharma \\ FET, Mody University \\ Lakshmangarh \\ Rajasthan, India
}

\author{
Deepak Sethi \\ FET, Mody University \\ Lakshmangarh \\ Rajasthan, India
}

\author{
P. P. Bhattacharya \\ FET, Mody University \\ Lakshmangarh \\ Rajasthan, India
}

\begin{abstract}
The scattered nature and active topology of wireless sensor networks (WSN) have some particular requirements - reduced energy consumption and extended network's lifetime. The paper provides a brief introduction about the wireless sensor network including the widely adopted architecture of wireless sensor network and wireless sensor node. The paper also focuses in critical issues of wsn that includes energy per packet, lower energy consumption, average packet delay, energy spent per round, packet size, distance, time until first node dies. The paper focuses on hierarchical routing protocols which are based on network structure scheme and explains how neural networks are helpful in providing energy efficiency to wireless sensor networks.
\end{abstract}

\section{Keywords}

Artificial Neural Networks, Energy Efficiency, Routing Protocols, Wireless Sensor Networks

\section{INTRODUCTION}

A wireless sensor network is defined as a collection of lowpower, short-lived, multi-functional sensor nodes which are restricted in memory capacity and radio range [1]. These sensors are provided with wireless interfaces with which they can communicate with one another to form a network. A very important and of course a basic function of WSN is to monitor the target area for a long period of time. These sensors are deployed in unreachable areas where refilling the sensor energy is usually impossible, a key issue in wireless sensor network applications is conserving sensor energy and prolonging the network lifetime [2]. There are basically two types of wireless sensor network - Unstructured and Structured WSN [3].

\section{a. Unstructured WSN}

It is the one containing a large collection of tiny sensor nodes. The sensor nodes are deployed randomly in the area. The network is left unattended to perform monitoring and reporting functions. Network maintenance which includes detecting failures and managing connectivity are quite difficult in unstructured wireless sensor networks. Also, due to random deployment there are uncovered areas left in unstructured WSN.

\section{b. Structured WSN}

In this type of sensor networks, all the nodes are deployed in a pre-planned manner. These sensor nodes are placed at specific position, which helps in providing full coverage. Wireless sensor networks are used in wide variety of everyday life activities and services which includes tracking and monitoring of events in various areas. Some of its applications are military uses of sensors to detect enemy intrusion, Environment monitoring (for example - forest fire detection), patient monitoring in heath care sectors etc. Widely adopted architecture of wireless sensor network is shown in Figure 1. Sensor nodes are usually scattered in a sensor field, which is an area where the sensor nodes are deployed. When a large number of nodes are present to monitor a phenomenon in an area, great amount accuracy is obtained.

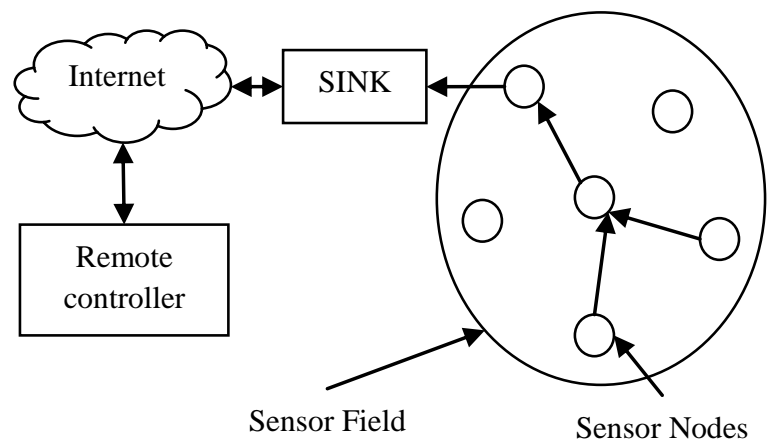

Figure 1: Architecture of Wireless Sensor Network.

Basically, a sensor node comprises of three basic components as illustrated Figure 2 - Sensing subsystem, Processing subsystem, Radio subsystem. These are used for data acquisition, local data processing and for wireless communication [4]. In addition a mobilizer - to change the location or configuration and a location finding subsystems to find the location of sensors are also present. A power unit is also attached. Sensor nodes coordinate among themselves to produce high-quality information about the physical environment. The decision of the sensor nodes for transmitting the data is based on the information it currently has and also on the knowledge of its computing, energy resources left and communication. Each of these sprinkled sensor nodes has the capacity to collect and route data either to other sensors or back to an external BS. 


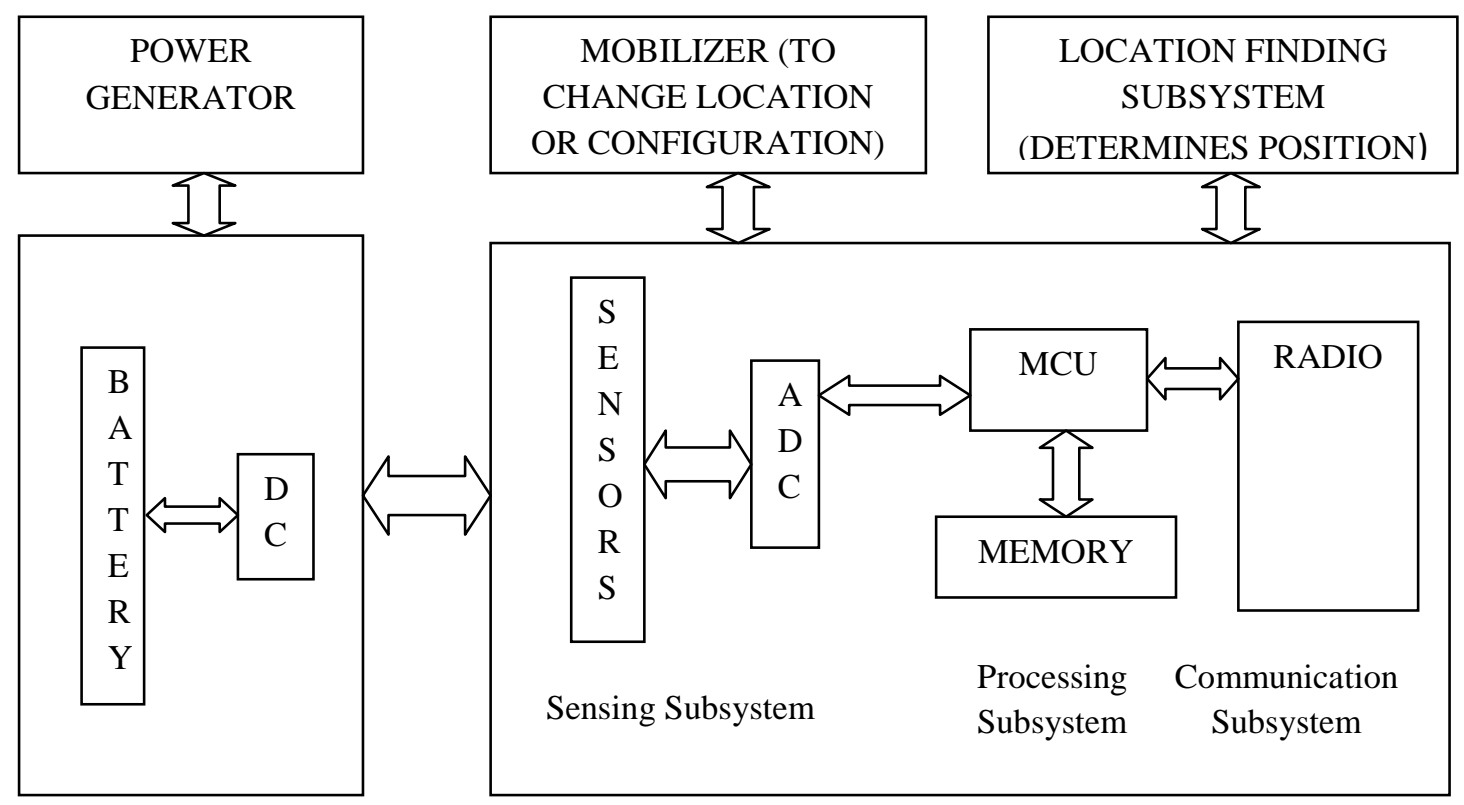

Figure 2: Architecture of Wireless Sensor Nodes.

\section{CRITICAL ISSUES OF WSN}

The main design goal of WSN is to not only transmit the data between source and destination but is also to increase the network lifetime; which can achieved by applying energy efficient protocols. The task of the protocol is not only to choose a path having lower energy consumption between sources to destination (BS), but also to find an efficient approach to prolong the network lifetime. But sometimes continuous use of low energy path may lead to energy depletion of sensor nodes in that path leading to network partition. Performance of the routing protocol is calculated based on some terms which includes [1]:-

a. Energy per Packet - The amount of energy required sending a packet from source to destination safely.

b. Low Energy Consumption - It includes such protocols that considers the remaining energy level of nodes and selects route accordingly for longevity of the network.

c. Total Number of Nodes Alive - It gives an idea of the area coverage of the network over time. This metrics is related to network lifetime.

d. Average Packet Delay - This metrics measure the accuracy of packet. This metrics is calculated as one way latency which is observed between transmission and reception of data packet at the sink.

e. Time until the First Node Dies - This metric indicates the duration for which all the sensor nodes on the network are alive. There are protocols in which the first node on the network runs out of energy earlier than in other protocols, but manages to keep the network operational much longer.

f. Energy Spent per Round - This metric is related to the total amount of energy spent in routing messages in a round. It is a short-term measure designed to provide an idea of the energy efficiency of any proposed method in a particular round. g. Packet Size - The lifetime of the network also depends on the packet size; it determines the time that a transmission will last. As it is effective in energy consumption so packet size should be reduced by combining large number of packets.

$h$. Distance - The distance between the transmitter and receiver can affect the power that is required to send and receive packets. The routing protocols can select the shortest paths between nodes and reduce energy consumption.

\section{ROUTING PROTOCOLS IN WSN}

Routing in wireless sensor networks is more difficult these days due to following reasons [1]. First, Sensor node requires careful supervision of resources. Second, wireless sensor networks are quite operation specific. Third, position of sensor nodes should be known at the time of data collection. Fourth, Data collected at the base station has higher possibility of redundancy. Due to such disparities, various routing algorithms have been developed. It has been said that protocols having higher energy efficiency will improve the longevity of network. The routing protocols can be classified into four main schemes [4]: Network Structure Scheme - the protocols are here classified on the basis of uniformity amongst nodes, Communication Model Scheme - the protocols are here classified on the basis of how the data is routed towards the base station in packets, Topology Based Scheme - the nodes here has to maintain the topological information of the wireless sensor network, and Reliable Routing Scheme - the protocols are here classified as how reliable a protocol is in terms of route failures. Our focus is on network structure scheme.

\subsection{Network Structure Scheme}

The protocols based on network structure can be classified on the basis of node uniformity. The nodes when are deployed uniformly over an area to form a network. It depends on networks whether they consider that all the nodes are equal to each other, while some other networks make distinction between different nodes of the network. More specifically, the main characteristic of the routing protocols that fit in to this category, it is the way that the nodes are connected and they 
route the information based on the networks architecture. This class includes flat and hierarchical protocols.

\subsubsection{Flat Protocols}

All the nodes in the network play the similar role. Flat network architecture presents several advantages, including minimum overhead to preserve the infrastructure between communicating nodes. It is divided into Pro-active and Reactive protocols.

\subsubsection{Pro-active Protocols}

These protocols are similar to wired network. The nodes periodically exchanges data between themselves and generate a routing table which is used to find the path to destination. These tables respond to the changes in the network by sending updates throughout the network. The route is discovered initially; thus no extra delay due to route discovery at the time of packet forwarding.

\subsubsection{Re-active Protocols}

It is also called source-initiated routing. The protocols in this category start route discovery procedure when required. When a route from source to destination is needed a global search procedure starts, this process causes delay as the routes are not available and have to be found. Sometimes routes are found in caches maintained by the sensor nodes.

\subsubsection{Hierarchical Protocols}

It is also called Cluster Based Routing [4]. The routing protocols in this category force a structure on the wireless sensor networks to provide energy efficient communication and scalability to the network. The network nodes are organized into clusters and based on some criteria a cluster head for each cluster is selected i.e. higher energy nodes are used to route the data, and lower energy nodes are used to sense the area. Figure 3 illustrates the clustering based scheme of the wireless sensor network. The cluster heads are then responsible for organizing the activities within the cluster. Here, in hierarchical routing the number of messages that are transferred to the base-station are decreased due to fusion and data aggregation thus it lowers the energy consumption and hence helps to improve the lifetime of the network. In such protocols the nodes around the base station and cluster head will exhaust their energy faster than all the other nodes. Some of the well known protocols under this section are: -

\section{a. Low Energy Adaptive Clustering Hierarchy} $(L E A C H)$ - LEACH is a clustering based algorithm. Key features of LEACH are: - First, to reduce the global communication it uses local compression. Second, it uses randomized rotation for making clusters. Third, for cluster setup and other operations localized coordination and control is required. Unlike, the conventional clustering algorithm, LEACH is self-organizing, adaptive clustering algorithm [6]. To distribute the energy load amongst the sensor it uses a process of randomization. In LEACH protocol the nodes of the network first organize themselves into clusters, where one node is elected as cluster head based on some criteria for coordinating activities and aggregating data that are to be sent to the base station.

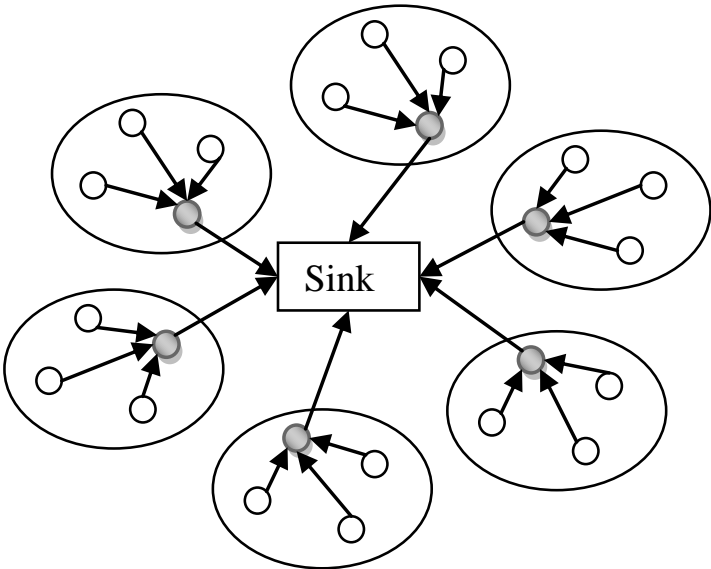

Figure 3: Clustering Schema for Wireless Sensor Networks.

The purpose of using randomized rotation for selecting cluster heads is -as in conventional clustering algorithm number of clusters are fixed, and the unlucky sensor nodes chosen as cluster head will die or deplete their energy quickly. Now the criteria based on which the nodes are selected as cluster head is based on a predefined probability [7]. These sensor nodes broadcast messages to other nodes of the network. The non cluster head nodes have to decide to which cluster it wants to belongs by choosing the cluster heads which require less energy for communication. Once the nodes are organized into clusters each cluster head creates a schedule for all the nodes belonging to its cluster. This schedule helps all the non cluster head nodes to be turned off all the time except at its transmission time thus helping to reduce the consumption of energy. Also, the nodes that have become the cluster head drain their battery a little fast. So, to spread the energy usage the cluster head are changed in every round.

The operation of LEACH protocols are broken into rounds and each round is divided into two phases the setup phase where the clusters are organized and steady state phase when actually the data is transferred to the base station [8].

SETUP PHASE - At this phase, adaptive clusters are created. At this point of time all the sensor nodes decides whether to become a cluster head for this particular round based on the two things. First - probability factor that is pre-determined; and Second - how many times the node has become cluster head so far.

The sensor node $\mathrm{n}$ has to choose a random number between 0 and 1. Based on the threshold condition presented in eq (1) the node is able to become cluster head for the current round [9].

$$
T(n)= \begin{cases}\frac{p}{1-p *\left(r \bmod \frac{1}{p}\right)} & \text { for } n \in G \\ 0 & \text { otherwise }\end{cases}
$$

Where

$p \rightarrow$ Is the percentage of cluster head (default value $=0.5$ ), $G \rightarrow$ Is the set of nodes that have not become cluster head for the last $\frac{1}{p}$ rounds,

i.e. when the random number chosen by the node is less than or equals to $\mathrm{T}(\mathrm{n})$ and $\mathrm{n} \in \mathrm{G}$ than the node will become cluster head. Following the condition every node will become cluster 
head at some point within $\frac{1}{p}$ rounds. At the very first round all the nodes are eligible to become cluster head, and the nodes that have become cluster head for the first round are not chosen cluster head for next $\frac{1}{p}$ rounds, it means other nodes have increased probability of becoming cluster heads.

Each node that has been elected as cluster head for the current round broadcast a message to the rest of the nodes. Cluster head uses CSMA-MAC protocol, and transmit their advertisement with the same transmission energy. Each non cluster head node has to decide to which cluster it should belong for the current round the decision is based on received signal strength of the advertisement i.e. the advertisement heard with the largest signal strength is the one to whom the minimum amount of transmitted energy is required for communication [9].

Next is all the nodes have to respond to the corresponding cluster heads that node will become a member of the cluster. CSMA-MAC protocol is again used by these non cluster head nodes to send this information back. Now, the cluster head nodes create a TDMA schedule to tell the non-cluster head nodes to tell when to transmit the data.

STEADY STATE PHASE - When the TDMA schedule is fixed the data transmission begins. It is assumed that the nodes always have data to send, they send it in the transmission time allotted to them. The radio of non-cluster head nodes can be turned off until the node's allocated transmission time. This helps in minimizing the energy dissipation of the network, hence improving the lifetime of the network. When the data from all the nodes is received by the cluster heads, it performs signal processing functions to compress the data into single signal. This composite signal is then sent to the base station.

\section{b. Low Energy Adaptive Clustering Hierarchy} Centralized (LEACH-C) - Since LEACH has a problem of determining the number of cluster heads in every round. For the formation of clusters LEACH-C utilizes the basestation [1]. In LEACH the nodes configure themselves to form clusters, but in LEACH-C at each iteration or round the basestation first receives the information about location, state and the energy level of the nodes. This information is used then to calculate the mean value of network node energy; and basestation finds predetermined number of cluster heads and hence configures the network into clusters [5]. The nodes that are selected as cluster head have higher energy level that the mean energy value. LEACH-C is an improvement over LEACH by the following points: First, to produce clusters that require less energy for transmission Base-Station utilizes it global knowledge of the network, Second, unlike LEACH where the number of cluster head varies from round to round due to lack of global coordination amongst nodes, in LEACH$\mathrm{C}$ the number of cluster heads in each round equals a predetermined optimal value [1].

\section{NEURAL NETWORK AND ENERGY CONSERVATION IN WSN}

A neural network is a massively parallel distributed processor made up of simple processing units which have a natural tendency for storing experiential knowledge and making it available for use [11]. A neural network resembles to brain in two aspects: First, knowledge is acquired by the network from its environment using a learning process. Second, Interneuron connection strength also known as synaptic weights is used to store the acquired knowledge. In other words, Artificial Neural Networks are arithmetic algorithms which are able to learn complicated mapping between input and output via a learning process or a supervised training, or they can even classify the input in an unsupervised manner.

Different types of training rules are applied to determine the way artificial neural network learns an input-output mapping. While we using neural network Learning is based on examples in which a set of correct input and output data is provided to the network and using these values the network learns by changing the weight values. These networks are then tested by providing a new sample, when the network returns correct answers as output [12]. We consider that the network has learnt. There are different types of network topologies, each of which has different capabilities according to the application. Prediction, Classification, and identification are some of the important application. Now the question is how neural networks help in energy conservation of wireless sensor networks. In fact, neural networks is not an energy conservation method, and even it cannot help independently to conserve the energy but it can be used with energy conservation methods as intelligent tools to provide better results and to work in more efficient, desirable and easier way. Thus for reducing the energy consumption in a wireless sensor network we can use neural networks with the same previous methods [13].

As it is said before to reduce the energy consumption we have to use energy efficient protocols only, but we can use neural network to get better result there are different roles a neural network can play in reducing the energy consumption as explain in [14] that it is quite necessary to find the exact positions of node for the efficient routing of packets and location aware services. The technique used to find the exact location of nodes is time difference of arrival (TDOA) with artificial neural network to find the exact location of nodes. TDOA is here used to calculate the distance between anchor node and sensor node by sending radio frequency signal and ultra sound signal, then sensor node note down the current time of both the signals. As the distance between the anchor node and the sensor node are available a neural network is created and is trained with these distance values and finds the approximate location coordinates of the system. The paper uses two types of neural network for training the WSN. First, Radial Basis Network Function, Second, Multilayer perceptron - it contains 3 Layers Input, Hidden and Output. In the different scenarios both the type of neural network provides better results than another. The error is also calculated between the exact position and the randomized position to provide the better results and to increase the network lifetime. WSN is used to monitor the interested region using multi-hop transmission, and coverage is the primary metrics to evaluate the monitoring capacity. Also the connectivity should be guaranteed so the BS is able to receive all the sensed data for further processing. Nodes have processing and communication capabilities which are used to collect the information and transmit it to the base station. Then the BS analyses and decides whether or not an exceptional event occurring in the network. Coverage and connectivity aware neural network based energy efficient routing protocol in WSN is explained in [15] a two layer feedforward neural network that implements the idea of competitive learning, where the sensor nodes of each cluster are provided as input to the neural network and the output of 
the network is node that is chosen as cluster head i.e. the node having minimum $\mathrm{E}_{\mathrm{id}}$ value. As the communication becomes the largest part of energy consumption for a wireless sensor node. A neural network based approach is explained in [16], the use of classification techniques of neural networks to reduce the data traffic from the node and hence reducing the energy consumption. Wireless sensor network is highly data centric. Data communication in WSN must be efficient one and must consume minimum power. Thus every sensor node is a source of data. The sensor data is classified using ART1 neural network model. It is an unsupervised neural network model which is used for classification of sensor data. ART1 classifies binary valued data; similarly Fuzzy ART is used to classify the input data that is fuzzy valued. Thus this technique ensures the uniform energy dissipation of all the nodes in the environment.

\section{CONCLUSION}

Energy conservation and increased network lifetime are the basic requirements of a wireless sensor network. To transmit data between sensor nodes routing protocols are used which can use single hop or multi-hop routing. These routing protocols try to minimize the energy consumption of the network. The performance of routing protocols is measured in terms of energy per packet, lower energy consumption, average packet delay, energy spent per round, packet size, distance, time until first node dies. Neural networks acquire knowledge via a learning process. Neural Networks can be easily used in wireless sensor network to increase the network lifetime. But neural network is not an energy conservation method but can be used with energy efficient routing protocols to improve performance of the wireless sensor network. There are various applications of neural network in wireless sensor network which include path discovery, sensor data classification, data prediction and node clustering.

\section{REFERENCES}

[1] Pantazis N. A., Nikolidakis S. A., Vergados D. D., "Energy-Efficient Routing Protocols in Wireless Sensor Networks: A Survey," IEEE Communications Surveys \& Tutorials, 2013, Vol. 15, Issue. 2, pp. 551-590.

[2] Akyildiz I. F., Su W., Sankarasubramaniam Y., Cayirci E., "Wireless sensor networks: a survey," ELSEVIER Computer Networks (38), 2002, pp. 393-422.

[3] Yick J., Mukherjee B., Ghosal D., "Wireless sensor network survey," Computer networks, 2008, Vol. 52, Issue. 12, pp. 2292-2330.

[4] Al-Karaki J. N., Kamal A. E., "Routing techniques in wireless sensor networks: a survey," Wireless communications, IEEE, 2004, Vol. 11, Issue. 6, pp. 6-28.

[5] Rahmanian A., Omranpour H., Akbari M., Raahemifar $\mathrm{K}$. "A novel genetic algorithm in LEACH-C routing protocol for sensor networks," In Electrical and Computer Engineering (CCECE), 2011 24th Canadian
Conference on IEEE, pp. 001096-001100.

[6] Saraswat J., Rathi N., Bhattacharya P.P., "Techniques to enhance the lifetime of wireless sensor network: A Survey," Global Journal of Computer Science and Technology, 2012, Vol. 12, Issue.14-E.

[7] Nikolidakis S. A, Kandris D, Vergados D. D, Douligeris $\mathrm{C}$, "Energy efficient routing in wireless sensor networks through balanced clustering." Algorithms, 2013, Vol.6, Issue. 1, pp. 29-42.

[8] Sindhwani N., Vaid R., "VLEACH: An Energy Efficient Communication Protocol for WSN," Mechanica Confab, 2013, Vol. 2, Issue. 2, pp. 79-84.

[9] Heinzelman W., Chandrakasan A., Balakrishnan H., "Energy-Efficient Communication Protocol for Wireless Microsensor Networks," In Proc. 33rd Hawaii International Conference on System Sciences, HI, USA, 2000, Vol. 8, pp. 110.

[10] Sharma, S., Mittal P., "Wireless Sensor Networks: Architecture, Protocols," International Journal of Advanced Research in Computer Science and Software Engineering, 2013, Vol. 3, Issue. 1, pp. 125-138.

[11] Sharma V., Rai S., Dev A., "A Comprehensive Study of Artificial Neural Networks", International Journal of Advanced Research in Computer Science and Software Engineering, 2012, Vol. 2, Issue 10, pp. 278-284.

[12] Maind S. B., Wankar P., "Research Paper on Basic of Artificial Neural Network", International Journal on Recent and Innovation Trends in Computing and Communication, 2014, Vol. 2 Issue 1. Pp 96-100.

[13] Enami N., Moghadam R. A., Dadashtabar K., Hoseini M., "Neural network based energy efficiency in wireless sensor networks: A survey," International Journal of Computer Science \& Engineering Survey, 2010, Vol. 1, Issue 1, pp. 39-53.

[14] Satyamurti S. K., Joshi R., "ANN Assisted Node Localization in WSN using TDOA," International Journal of Innovative Research in Computer and Communication Engineering, 2014, Vol. 2, Issue 4, pp. 3871-3877.

[15] Kumar N., Kumar M., Patel R. B., "Coverage and Connectivity Aware Neural Network Based Energy Efficient Routing in Wireless Sensor Networks," Journal on Application of Graph Theory in Wireless Ad-hoc Networks and Sensor Networks, 2010, Vol. 2, Issue 1,pp. 45-60.

[16] Akojwar S. G., Patrikar R. M., "Improving Life Time of Wireless Sensor Networks Using Neural Network Based Classification Techniques with Cooperative Routing," International Journal of Communications, 2008, Vol.2, Issue 1, pp. 75-86. 